\title{
Iterative Compilation Optimization Based on Metric Learning and Collaborative Filtering
}

\author{
HONGZHI LIU, School of Software and Microelectronics, Peking University, P.R. China \\ JIE LUO, Center for Data Science, Peking University, P.R. China \\ YING LI, National Engineering Center of Software Engineering, Peking University, P.R. China \\ ZHONGHAI WU, National Engineering Center of Software Engineering, Peking University, \\ P.R. China
}

Pass selection and phase ordering are two critical compiler auto-tuning problems. Traditional heuristic methods cannot effectively address these NP-hard problems especially given the increasing number of compiler passes and diverse hardware architectures. Recent research efforts have attempted to address these problems through machine learning. However, the large search space of candidate pass sequences, the large numbers of redundant and irrelevant features, and the lack of training program instances make it difficult to learn models well. Several methods have tried to use expert knowledge to simplify the problems, such as using only the compiler passes or subsequences in the standard levels (e.g., -O1, -O2, and -O3) provided by compiler designers. However, these methods ignore other useful compiler passes that are not contained in the standard levels. Principal component analysis (PCA) and exploratory factor analysis (EFA) have been utilized to reduce the redundancy of feature data. However, these unsupervised methods retain all the information irrelevant to the performance of compilation optimization, which may mislead the subsequent model learning.

To solve these problems, we propose a compiler pass selection and phase ordering approach, called Iterative Compilation based on Metric learning and Collaborative filtering (ICMC). First, we propose a data-driven method to construct pass subsequences according to the observed collaborative interactions and dependency among passes on a given program set. Therefore, we can make use of all available compiler passes and prune the search space. Then, a supervised metric learning method is utilized to retain useful feature information for compilation optimization while removing both the irrelevant and the redundant information. Based on the learned similarity metric, a neighborhood-based collaborative filtering method is employed to iteratively recommend a few superior compiler passes for each target program. Last, an iterative data enhancement method is designed to alleviate the problem of lacking training program instances and to enhance the performance of iterative pass recommendations. The experimental results using the LLVM compiler on all 32 cBench programs show the following: (1) ICMC significantly outperforms several state-ofthe-art compiler phase ordering methods, (2) it performs the same or better than the standard level -O3 on

This work was partially sponsored by Key R\& Project of Guangdong Province (No. 2020B010164003), National Natural Science Fund of China (Grant No. 61232005) and Delta Innovation Research Program.

Authors' addresses: H. Liu (corresponding author), School of Software and Microelectronics, Peking University, No. 24 Jinyuan Road, Beijing, P.R. China; email: liuhz@pku.edu.cn, J. Luo, Center for Data Science, Peking University, No. 5 Yiheyuan Road, Beijing, P.R. China; email: isluojie@pku.edu.cn, Y. Li, National Engineering Center of Software Engineering, Peking University, No. 5 Yiheyuan Road, Beijing, P.R. China; email: li.ying@pku.edu.cn, Z. Wu (corresponding author), National Engineering Center of Software Engineering, Peking University, No. 5 Yiheyuan Road, Beijing, P.R. China; email: wuzh@pku.edu.cn.

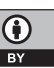

This work is licensed under a Creative Commons Attribution International 4.0 License.

(C) 2021 Association for Computing Machinery.

1544-3566/2021/12-ART2 \$15.00

https://doi.org/10.1145/3480250 
all the test programs, and (3) it can reach an average performance speedup of 1.20 (up to 1.46) compared with the standard level -O3.

CCS Concepts: • Software and its engineering $\rightarrow$ Compiler; • Information system $\rightarrow$ Recommender systems;

Additional Key Words and Phrases: Compiler auto-tuning, iterative compilation, metric learning, collaborative filtering

\section{ACM Reference format:}

Hongzhi Liu, Jie Luo, Ying Li, and Zhonghai Wu. 2021. Iterative Compilation Optimization Based on Metric Learning and Collaborative Filtering. ACM Trans. Arch. Code Optim. 19, 1, Article 2 (December 2021), 25 pages. https://doi.org/10.1145/3480250

\section{INTRODUCTION}

The goal of compilers is to translate program codes written by humans into executable binaries for efficient execution by a target computing hardware. For each program, there are many different correct translations having varying performance characteristics. The key is to find the best one among these correct translations [45]. The selection of a sequence of suitable compiler passes for a given program has usually been done manually [5]. However, the increasing number of candidate compiler passes and diverse hardware architectures make it difficult to do this by hand.

Modern compilers provide a large number of optimization passes targeting various code segments. For example, GCC has more than 200 compiler passes [32], and LLVM has over 150 transformation passes [5]. Most of these compiler passes are disabled by default. Compiler designers assume software developers know which compiler passes will be beneficial for their programs. To simplify the problem, compiler designers always provide several standard optimization levels, such as $-\mathrm{O} 1,-\mathrm{O} 2$, and $-\mathrm{O} 3$. Each of these standard levels is a fixed and program-independent sequence of compiler passes. However, these standard sequences leave much to be desired performance for most programs [2, 24, 38]. Many experiments have verified that the performance of compilers' standard levels is not good enough for specific benchmarks and that the best pass sequence is program-specific [6,31]. For each program, choosing the right set of compiler passes (aka pass selection) and finding the best ordering of them (aka phase ordering) are two critical problems for compiler auto-tuning [6, 24, 25].

Recent research efforts have attempted to utilize machine learning models to deal with these two NP-hard problems, pass selection and phase ordering [45]. However, the large search space of candidate pass sequences, the large numbers of redundant and irrelevant features, and the lack of training program instances make it difficult to learn models well $[12,13]$. To deal with the problem of large search space, several studies have tried to use expert knowledge to prune the search space of candidate pass sequences [8, 41, 43]. For example, Ashouri et al. [3, 4] used compiler designers' knowledge to construct pass subsequences from a standard optimization level and then utilized the subsequences as basic optimization units to form the search space. These methods can greatly prune the search space. However, they are limited to the passes and their orderings used in the standard level while ignoring other useful passes. To solve this problem, we propose a datadriven method to construct pass subsequences. It tries to mine the collaborative interactions and dependency among passes according to the collected performance records of candidate passes and pass-pairs on a given set of programs. Therefore, all available passes provided by compilers can be utilized to further optimize programs. In addition, it can help us to discover the interactions and dependency among passes that have been ignored by experts and to further design new standard optimization sequences for compilers. 
To deal with the problem of large numbers of redundant and irrelevant features, principal component analysis (PCA) [1, 12, 44] and exploratory factor analysis (EFA) [6] have been utilized for compiler auto-tuning. These unsupervised methods are good at removing the redundancy of feature data. However, all the irrelevant information, for example, the number of indirect pointers and the number of occurrences of constant 1 , is retained because these methods ignore the effects of features on the performance of compilation optimization, which may mislead the subsequent model learning. To solve this problem, we utilize a supervised metric learning method, which tries to retain useful feature information for compilation optimization while removing both the irrelevant and the redundant information. Based on the learned similarity metric, a neighborhoodbased collaborative filtering method is designed to iteratively recommend a few superior compiler passes for each target program. In addition, a data enhancement method is designed to alleviate the problem of lacking training program instances.

The main contributions of this article are summarized as follows:

(1) We propose a data-driven method to construct pass subsequences according to the observed collaborative interactions and dependency among passes on a given program set. Therefore, we can make use of all available passes while reducing the search space. In addition, it can find out some collaborative interactions ignored by experts.

(2) We propose an iterative compilation optimization method based on metric learning and collaborative filtering, which tries to recommend passes for the target program according to the observed preferences of similar programs. It utilizes a supervised metric learning method to embed programs into a latent representation space with the supervision of their preference similarities on candidate pass subsequences, which can remove both the irrelevant and the redundant information in program features for compilation optimization.

(3) An iterative data enhancement method is designed to alleviate the problem of lacking training program instances and to enhance the performance of iterative pass recommendations.

(4) We evaluate the proposed method and make a comparison with several state-of-the-art compiler phase ordering methods on all $32 \mathrm{cBench}$ programs. Extensive experiments demonstrate the promise of our method, which performs the same or better than the standard level -O3 on all the test programs and can reach an average performance speedup of 1.20 (up to 1.46).

The rest of this article is organized as follows: Section 2 introduces some related work. Section 3 formulates the problem and Section 4 describes our method in detail. Comparison with several state-of-the-art methods and empirical evaluations are presented in Section 5. Finally, Section 6 concludes the article.

\section{RELATED WORK}

\subsection{Reduction of Search Space}

The main difficulty of compiler pass selection and phase ordering is the large and intractable search space caused by hundreds of candidate passes. Evolutionary algorithms (EAs), such as genetic algorithms (GAs) and genetic programming (GP), have been used to find good optimization solutions (pass sequences) from the large search space for each given program [5]. Cooper et al. $[15,16]$ proposed to use a GA to find the pass sequences that can generate smaller object codes to reduce the code size for embedded systems. Kulkarni et al. [30] used two complementary GAs to shorten the search time by pruning unnecessary optimization configurations and to increase the variety of choices. Stephenson et al. [42] proposed to use GP to heuristically explore the space. EAs are useful for exploring the large search space. However, they rely on excessive fitness evaluations and expensive exploration time [32, 45]. 
To reduce the search space, several methods have been proposed to construct pass subsequences and search the best whole sequence based on these subsequences [28]. Ashouri et al. [3] directly took some fragments of a standard level provided by compiler designers as subsequences. Later, Ashouri et al. [4] proposed to cluster the passes used in a standard level into several groups with a hierarchical clustering algorithm and combine each group as a subsequence. These methods can greatly reduce the search space. However, they depend on the information of expert-designed pass sequences. In addition, they ignore other useful passes that are not contained in the standard level.

\subsection{Machine Learning Based Sequence Prediction}

To avoid excessive online evaluations for each target program, several machine learning-based methods have been proposed to predict the optimization effects of candidate pass sequences on each given program. These methods can be roughly divided into two categories according to the search space exploration strategy, namely (1) complete sequence prediction and (2) iterative intermediate prediction. The former strategy tries to directly predict the global best pass sequence for a given program based on the characteristics of the program in its original state $[4,10,39,40]$. The latter strategy tries to iteratively predict the next-best pass to further optimize the given program based on the characteristics of the program in its current optimized state and/or performance feedback $[1,6,32,33]$.

Ashouri et al. [4] proposed to learn a prediction model that takes a tuple $(F, T)$ as an input, where $F$ denotes the code-feature vector of the program being optimized and $T$ denotes one of the possible compiler pass sequences. A similar machine learning-based method [10] was proposed for pass selection. These methods can greatly reduce the requirement of online evaluations using models learned offline. However, the code features extracted by different tools contain much redundant and irrelevant information for compilation optimization, which makes it difficult for model learning. In recent years, several reaction-feature based learning methods have been proposed for compiler auto-tuning. Cereda et al. [11] assumed that if two programs receive similar performance improvements from the same set of passes, they will show similar reactions on other sets. Furthermore, they proposed a collaborative filtering-based method to deal with the pass selection problem. This method can be extended for phase ordering problem by regarding each optimization configuration as a complete pass sequence rather than a pass set. Christoforidis et al. [14] proposed to use a matrix factorization based method to learn a regression model according to collected reaction data to deal with the phase ordering problem. These methods can avoid the problems of redundant and irrelevant code-features. However, they require several online evaluations to characterize each new program and make predictions because of ignoring the useful information contained in code features, which makes them heavy time-overhead during the online compilation phase.

In contrast to complete sequence prediction methods that try to directly optimize each whole program, intermediate prediction methods try to iteratively optimize different segments of a given program according to the current state of the program [3, 5, 29,32]. They use an iterative process to select the next-best compiler pass based on the current intermediate state of the given program, i.e., the state of the program optimized by several previously selected passes. At each intermediate step, they only require evaluating or predicting the performance of all candidate passes instead of all candidate pass sequences, which greatly reduces the computational complexity.

Kisuki et al. [29] treated the compilation optimization as an iterative tree-based search process. The next-best compiler pass is predicted for the current optimized program according to the program's feedback. Therefore, it can iteratively generate further efficient codes. However, this method requires all candidate passes to be evaluated at each sequence node, which is expensive for general compilation. In addition, it ignores the interactions among passes, which is important and significant for optimization effectiveness and efficiency of phase ordering. 
Ashouri et al. [3] proposed to use a linear regression model to predict the intermediate speedup value of candidate compiler optimizations and select the next-best optimization given the current state of the program. It avoids the evaluations of all candidate passes at each intermediate state. In addition, it tries to capture the interactions among passes by using subsequences of standard level -O3 as the candidate optimizations. However, this method ignores other useful compiler passes outside of -O3. In addition, it only makes use of dynamic features to characterize programs while ignoring useful static code-features.

\subsection{Data Transformation and Enhancement}

The existence of large numbers of redundant and irrelevant features and the lack of training program instances are two common problems for machine learning-based methods [35].

To deal with the problem of large numbers of redundant features, several data transformation methods have been utilized for compiler auto-tuning [6, 7, 44]. Principal component analysis (PCA) [3, 12, 44] and exploratory factor analysis (EFA) [6] are two commonly used data transformation methods [5]. They are good at removing the redundancy of feature data. However, these unsupervised methods only try to maintain the main characteristics of data while ignoring their effects on the performance of compilation optimization. Therefore, the information irrelevant to compilation optimization is also retained.

To deal with the problem of lacking program instances, several methods have been proposed to generate synthetic training programs. Chiu et al. [13] designed a programming language that allows users to annotate a template program to customize its code using statistical distributions and to generate program instances based on those distributions. Cummins et al. [18] tried to use deep learning techniques to automatically construct models for how humans write programs from open source repositories. Then, they synthesized programs through a process of iterative model sampling. These methods can generate more synthetic programs for model training. However, they rely on manual programming or a large source code repository.

\section{PROBLEM DEFINITION}

Let $O=\left\{o_{1}, o_{2}, \ldots, o_{m}\right\}$ denote a set of candidate compiler passes and $P=\left\{p_{1}, p_{2}, \ldots, p_{n}\right\}$ denote a set of programs, where $m=|O|$ and $n=|P|$ denote the number of passes and programs, respectively. Let $s_{j}=\left(o_{j 1}, o_{j 2}, \ldots, o_{j s}\right)$ denote a sequence of compiler passes and $\Omega$ denote the set of all possible pass sequences. Compilation optimization can be regarded as a function $G$. Its input is a program $p_{i}$ and a pass sequence $s_{j}$, based on which it outputs $p_{i}$ 's semantically equivalent code optimized by $s_{j}$, i.e.,

$$
p_{i}^{j}=G\left(p_{i}, s_{j}\right) .
$$

The goal of pass selection and phase ordering is to select the best sequence $s^{*} \in \Omega$ for each program $p_{i} \in P$, i.e.,

$$
s^{*}\left(p_{i}\right)=\max _{s_{j} \in \Omega} f\left(p_{i}, s_{j}\right),
$$

where $f\left(p_{i}, s_{j}\right)$ denotes the performance improvement of program $p_{i}$ with the help of pass sequence $s_{j}$.

The pass selection and phase ordering problem is an NP-hard problem. Let $L$ denote the maximum length of the pass sequence. The size of the search space is

$$
|\Omega|=\sum_{i=0}^{L} m^{i},
$$

where $m$ denotes the number of candidate passes. 


\section{THE PROPOSED METHODOLOGY}

The framework of the proposed pass selection and phase ordering model, called Iterative Compilation based on Metric learning and Collaborative filtering (ICMC), is shown in Figure 1. To alleviate the problem of a large search space caused by hundreds of candidate passes, we first propose a data-driven pass subsequence construction method. It makes use of the observed collaborative interactions and dependency among passes on the training program set to automatically generate a set of pass subsequences (Section 4.1). Then, we propose an iterative compilation method based on metric learning and collaborative filtering (Section 4.2). It iteratively recommends the next-best subsequence according to the current state of the target program and the performance of candidate subsequences on similar programs (Section 4.2.2). To avoid the misleading effects of both the irrelevant and the redundant features on similarity measurement, we utilize a supervised metric learning method to automatically learn an appropriate similarity metric for the subsequence recommendation task. It makes use of the observed preferences of training programs on candidate pass subsequences (Section 4.2.1). Furthermore,we propose a data enhancement method to deal with the problem of lacking training program instances for metric learning and subsequence recommendation (Section 4.2.3).

\subsection{Construction of Collaborative Pass Subsequences}

We assume that there are collaborative interactions among passes and that some of these interactions are useful for improving the performance of compilation optimization and controlling the search space [28]. For a given program $p$, if pass sequence $(A, B)$ performs better than both pass $A$ and pass $B$ alone and even better than the sequence $(B, A)$, i.e.,

$$
f(p,(A, B))>f(p,(A)) \& f(p,(A, B))>f(p,(B)) \& f(p,(A, B))>f(p,(B, A)),
$$

we say that pass $A$ improves the effectiveness of pass $B$ for program $p$, which means that pass $B$ depends on pass $A$. These kinds of relationships among passes are called collaborative interactions in this article.

The interactive effects of pass pairs depend on the characteristics of the programs being optimized. We assume that if one collaborative interaction exists on a vast number of programs, it is a strong collaborative interaction. Based on this assumption, we design a data-driven method to make use of all available passes besides those not contained in the standard levels.

Algorithm 1 describes the process of constructing pass subsequences according to the collaborative interactions among passes on a given program set $P$. First, we remove some inefficient passes to reduce the complexity of subsequence construction while excluding few potentially useful passes. Second, we collect collaborative pass pairs on all programs to construct a collaboration matrix $M$. The element $M_{i j}$ of matrix $M$ denotes the frequency of collaborative pass pair $\left(o_{i}, o_{j}\right)$ appearing in the program set $P$, where $o_{i}$ and $o_{j}$ denote the $i$ th and $j$ th pass, respectively. Third, we construct a dependency graph $G$ according to the collected collaboration matrix. The dependency graph $G$ is a directed weighted graph, whose nodes denote compiler passes. If there exist collaborative interactions between two passes $o_{i}$ and $o_{j}$, there is an edge between the two corresponding nodes in the graph $G$ and the weight of this edge is $M_{i j}$. Fourth, we utilize a graph cut algorithm [46] to cut down a few weak connections while keeping the strong collaborative connections. The graph is segmented into several subgraphs. Finally, each subgraph is stretched as a subsequence according to the collaborative connections inside it. Figure 2 shows an example of the graph cut process, which cuts down the weak connections and generates two strongly connected subgraphs. For each subgraph, we use a depth-first traversal algorithm to construct a corresponding pass subsequence. First, we find the root node of the subgraph according to the 


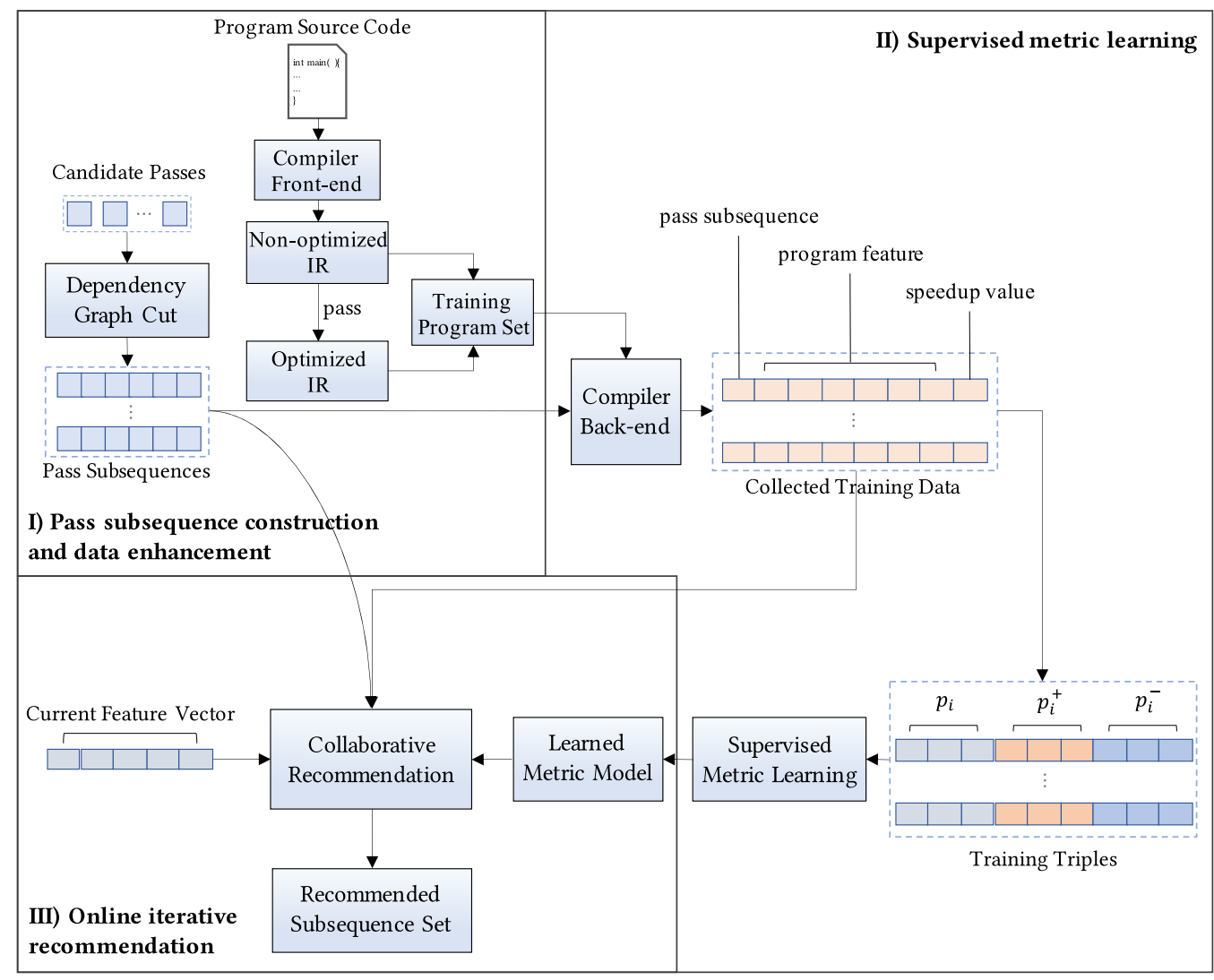

Fig. 1. Framework of the proposed ICMC model. It consists of three main components. I) Pass subsequence construction and data enhancement denotes the preprocessing phase, which aims to reduce the search space by subsequence construction and generate more training program instances by data enhancement. II) Supervised metric learning aims to automatically learn an appropriate similarity metric for the subsequence recommendation task. III) Online iterative recommendation aims to iteratively recommend the next-best pass subsequence according to the current feature state of the given program, the learned metric model, and the collected training data.

principle of minimum in-degree. Then, we recursively traverse the subtrees of the root which are ranked according to their weights connected to the root, i.e., $M_{i j}$. It ensures that the pass which can enhance the effectiveness of subsequent passes is used first.

\subsection{Iterative Compilation Based on Metric Learning and Collaborative Filtering}

The optimization passes provided by compilers are targeted for different code segments of programs, such as some for loop unrolling while some others for register allocation. Based on this fact, we assume that the same pass subsequence has similar effects on similar programs. Furthermore, we design a neighborhood-based collaborative filtering method to iteratively recommend pass subsequences for a given program (Section 4.2.2). The key of this method is to determine the neighborhood according to similarities between programs. However, similarities based on the original feature vectors of programs may mislead the recommendations because of the numerous redundant and irrelevant features. To deal with this problem, we utilize a supervised metric learning method to automatically learn an appropriate similarity metric for pass subsequence 


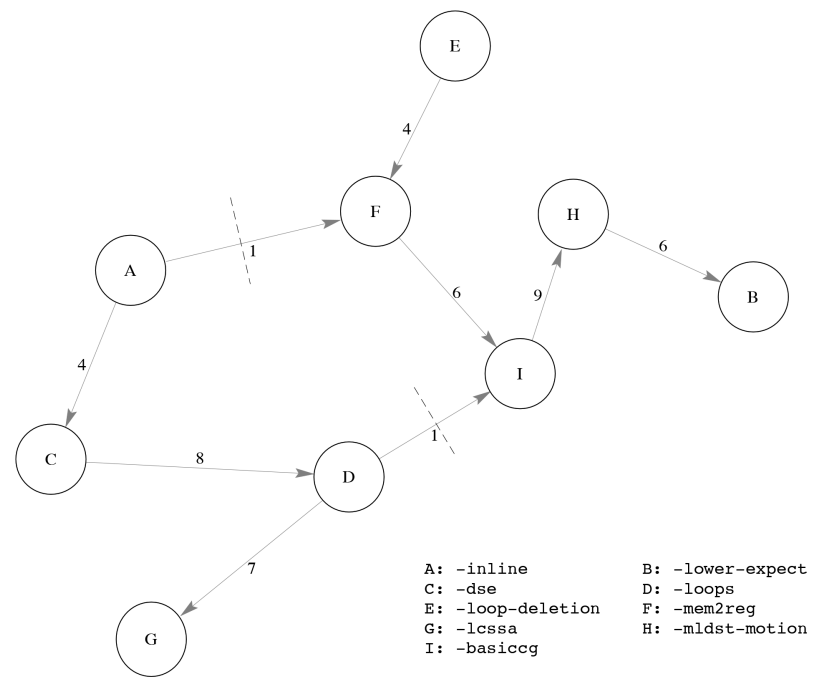

Fig. 2. An example of data-driven subsequence construction. Nodes of the graph denote compiler passes, arrows denote directed collaborative interactions, and weights of edges denote the frequencies of collaborative interactions that appear in the training program set. Two weak connections are cut down, and the constructed subsequences are as follows: A-C-D-G (-inline -dse -loops -lcssa) and E-F-I-H-B (-loop-deletion -mem2reg -basiccg -mldst-motion -lower-expect).

recommendation (Section 4.2.1). The performance of both metric learning and subsequence recommendation relies on a set of sufficient training samples. However, the available programs are always limited. To deal with this problem, we design a data enhancement method to generate more training programs based on the available programs and compiler passes (Section 4.2.3).

4.2.1 Program Similarity Based on Metric Learning and Programs' Preferences. We assume that similar programs have similar preferences on candidate pass subsequences with the same running environment [37]. To profile programs comprehensively, we make use of both dynamic and static features to characterize them. Dynamic features characterize the behavior of the target program when executing, while static features characterize the source code of the program without execution. However, because of the vastness and variety of features collected through different feature extractors, there may be a large number of redundant or irrelevant features for performance prediction of compilation optimization. Therefore, similarity based on the original program feature vectors may not satisfy our assumption. To deal with this problem, we utilize a supervised metric-learning framework [36] to embed the programs into a latent representation space with consideration of programs' preferences on candidate passes or subsequences.

As shown in Figure 3, in the learned latent representation space, we hope the program pair $\left(p_{i}, p_{i}^{+}\right)$with similar preferences on candidate pass subsequences becomes closer while the encounter pair $\left(p_{i}, p_{i}^{-}\right)$becomes more distant.

Let $\left(F_{i}, \Upsilon_{i}\right)$ denote the instance of program $p_{i}$, where $F_{i}$ denotes its feature vector and $\Upsilon_{i}$ denotes its preference vector on candidate pass subsequences. The feature vector $F_{i}$ contains both static features and dynamic features of program $p_{i}$, which are extracted by different tools, such as MICA [27] and Milepost GCC [22]. The preference vector $\Upsilon_{i}$ represents the performance improvement of program $p_{i}$ compiled with the candidate pass subsequences constructed in Section 4.1. Its element $\Upsilon_{i j}$ denotes the performance improvement of program $p_{i}$ after being optimized with the subsequence $s_{j}$, i.e., the speedup value $f\left(p_{i}, s_{j}\right)$. And the length of $\Upsilon_{i}$ is the number of 


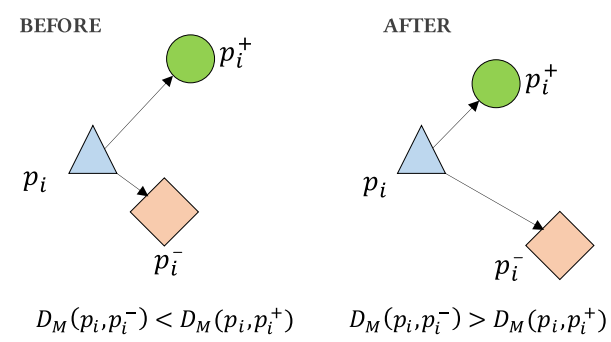

Fig. 3. Illustration of the supervised metric learning. The program $p_{i}^{+}$with similar preferences on candidate pass subsequences is pulled closer while the dissimilar program $p_{i}^{-}$is pushed away.

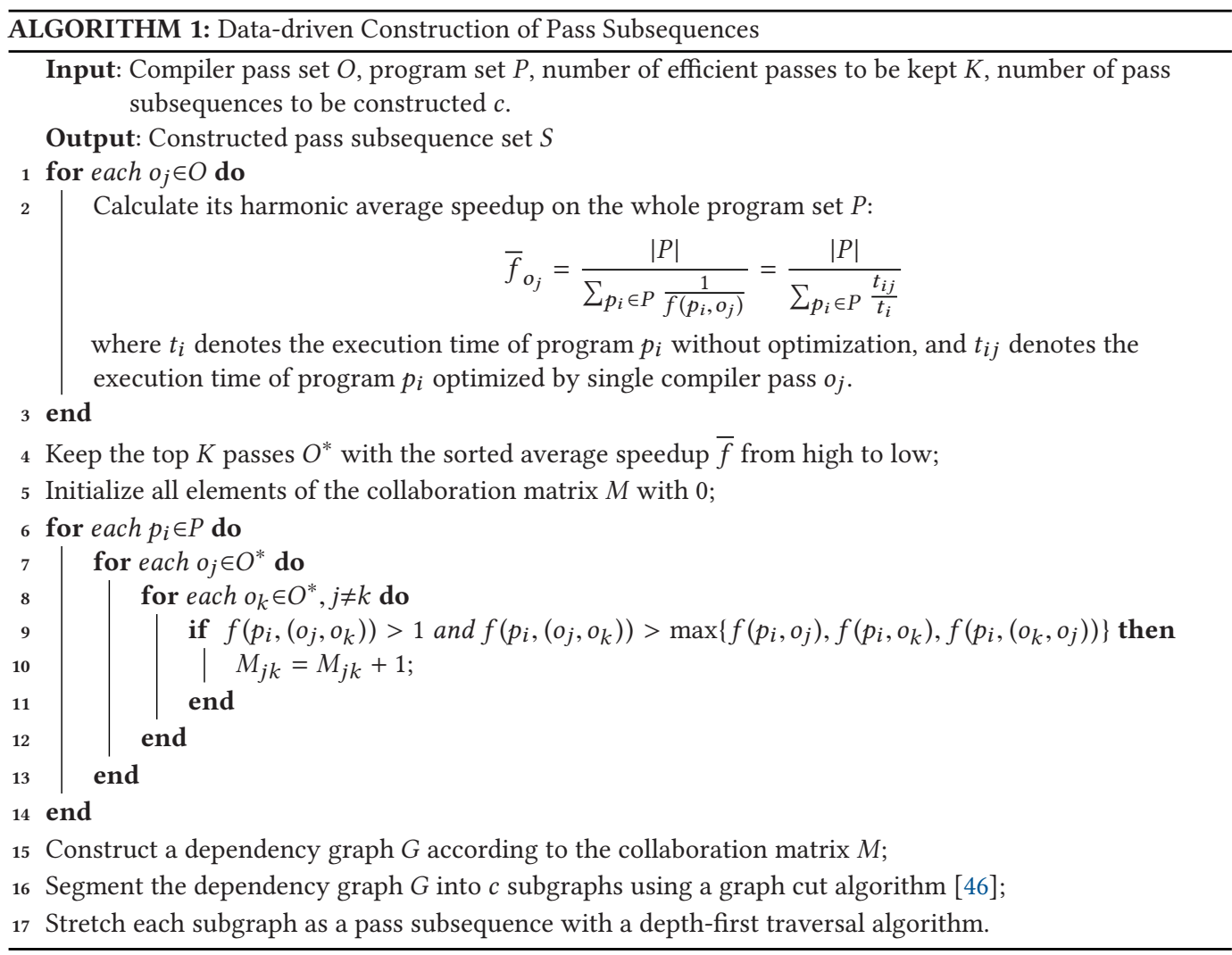

candidate subsequences. To learn the latent representations of programs with consideration of their preferences on candidate pass subsequences, we utilize the LSML model [36] and try to learn a Mahalanobis transformation matrix $M$ to minimize the following squared hinge loss function:

$$
\begin{gathered}
\operatorname{Loss}_{M L}=\sum_{\left(p_{i}, p_{i}^{+}, p_{i}^{-}\right) \in \text { TrainSet }} H\left(D_{M}\left(p_{i}, p_{i}^{+}\right)-D_{M}\left(p_{i}, p_{i}^{-}\right)\right)+D_{l d}\left(M, M_{0}\right), \\
H(x)= \begin{cases}0 & (x \leq 0) \\
x^{2} & (x>0)\end{cases} \\
D_{l d}\left(M, M_{0}\right)=\operatorname{trace}\left(M M_{0}^{-1}\right)-\log \operatorname{det}(M),
\end{gathered}
$$




$$
D_{M}\left(p_{i}, p_{j}\right)=\sqrt{\left(F_{i}-F_{j}\right)^{T} M\left(F_{i}-F_{j}\right)}
$$

where $D_{M}\left(p_{i}, p_{j}\right)$ represents the Mahalanobis distance between program $p_{i}$ and program $p_{j}$ after being embedded with the transformation matrix $M$, and $D_{l d}\left(M, M_{0}\right)$ represents the LogDet divergence between the learned metric $M$ and a given prior metric $M_{0}$ (usually set as the identity matrix). A triplet $\left(p_{i}, p_{i}^{+}, p_{i}^{-}\right)$represents that program $p_{i}^{+}$is more similar than program $p_{i}^{-}$for a given program $p_{i}$ according to their preferences on candidate pass subsequences. The preference similarity between two programs is computed as the cosine similarity of their preference vectors with respect to the candidate pass subsequence set $S$ :

$$
S_{\text {preference }}\left(p_{i}, p_{j}\right)=\frac{\sum_{s_{k} \in S} \Upsilon_{i k} \Upsilon_{j k}}{\sqrt{\sum_{s_{k} \in S} \Upsilon_{i k}^{2}} \sqrt{\sum_{s_{k} \in S} \Upsilon_{j k}^{2}}}
$$

where $\Upsilon_{i k}=f\left(p_{i}, s_{k}\right)$ represents the performance improvement of program $p_{i}$ with the help of candidate pass subsequence $s_{k}$. The more similarity there is between two programs, i.e., the more similar the candidate subsequences' speedup on the two programs, the closer the value $S_{\text {preference }}\left(p_{i}, p_{j}\right)$ is to 1 . To learn the transformation matrix $M$ given a set of programs $P$ with their instances $\left\{\left(F_{i}, \Upsilon_{i}\right) \mid p_{i} \in P\right\}$, we adopt a projected gradient method to optimize the objective function in Equation (5) as in [36].

With the learned metric $M$, we can calculate the similarity between programs to select the neighborhood for a new program according to Equation (10), which normalizes the similarity to the interval of $(0,1]$.

$$
\operatorname{sim}\left(p_{i}, p_{j}\right)=\frac{1}{1+D_{M}\left(p_{i}, p_{j}\right)}
$$

where $D_{M}\left(p_{i}, p_{j}\right)$ denotes the distance between program $p_{i}$ and program $p_{j}$ according to the learned metric model $M$ and their features $F_{i}$ and $F_{j}$ as in Equation (8).

4.2.2 Personalized Recommendation Based on Collaborative Filtering. Based on the learned similarity metric, we take each program as a user and design a neighborhood-based collaborative filtering method to iteratively recommend pass subsequences for a new given program. Figure 4 shows an example of this program-based collaborative filtering. As shown in the figure, for a new program $p_{\text {new }}$, the recommendation is made based on the preferences of its similar program $p_{1}$ and $p_{2}$ on candidate pass subsequences.

Assume we have collected a set of instances $I=\left\{\left(F_{i}, \Upsilon_{i}\right) \mid p_{i} \in P\right\}$ for a given program set $P$. Each instance $\left(F_{i}, \Upsilon_{i}\right)$, which contains a feature vector $F_{i}$ and a preference vector $\Upsilon_{i}$, corresponds to a program sample $p_{i} \in P$. The preference vector $\Upsilon_{i}$ contains the preferences of program $p_{i}$ on all candidate pass subsequences. The preference of program $p_{i}$ on a pass subsequence $s_{j}$ is measured by the performance improvement of program $p_{i}$ after being compiled with subsequence $s_{j}$, i.e., the speedup value $f\left(p_{i}, s_{j}\right)$.

The instances of the $k$ most similar programs in $P$ are used to recommend subsequences for a new given program $p_{\text {new }} \notin P$. The preference of program $p_{\text {new }}$ on a candidate pass subsequence $s_{j}$ can be estimated as follows:

$$
r\left(p_{\text {new }}, s_{j}\right)=\sum_{p_{v} \in N\left(p_{\text {new }}, k\right)} \operatorname{sim}\left(p_{\text {new }}, p_{v}\right) R_{v j}
$$

where $N\left(p_{\text {new }}, k\right)$ denotes the program set of the $k$ nearest neighbors of program $p_{\text {new }}$ in $P$, $\operatorname{sim}\left(p_{\text {new }}, p_{v}\right)$ represents the similarity between program $p_{\text {new }}$ and program $p_{v}$ as in Equation (10), and $R_{v j}$ represents the effectiveness of subsequence $s_{j}$ on program $p_{v}$. Considering the large deviation of subsequences' performance on programs, we take $R_{v j}$ as a binary variable to represent 


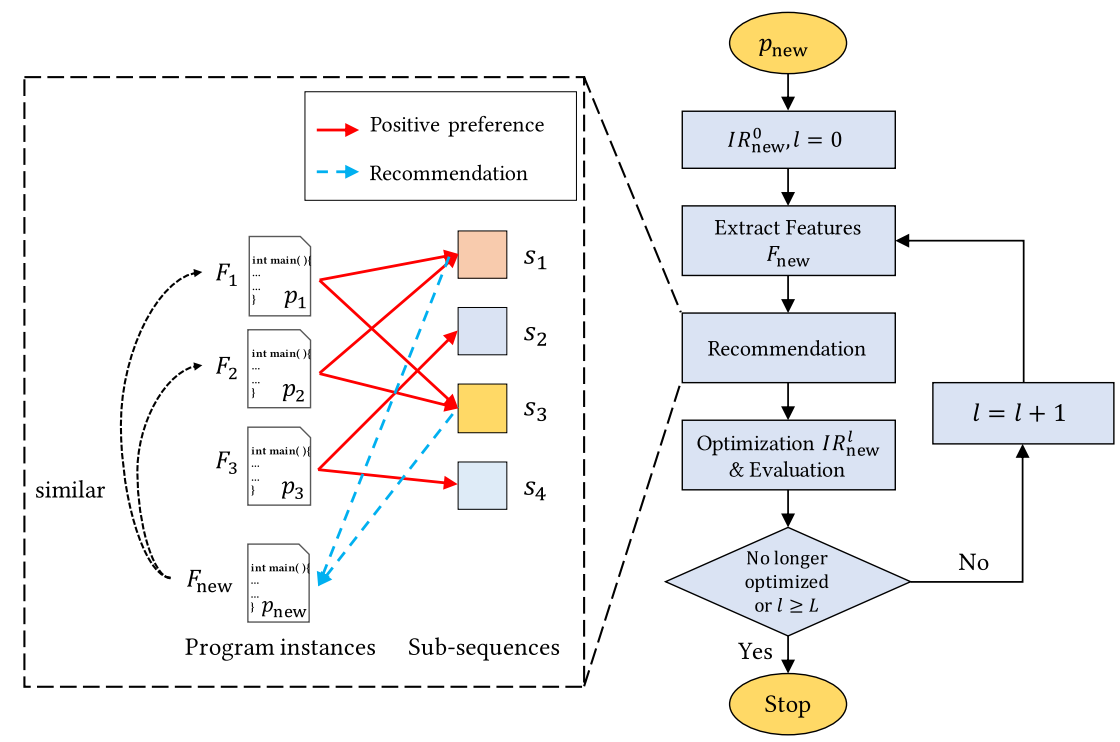

Fig. 4. Illustration of the iterative optimization process and an example of program-based collaborative filtering.

the interaction rather than the speedup value. The positive interaction $R_{v j}=1$ is defined such that the program $p_{v}$ compiled with the subsequence $s_{j}$ can obtain the top- $n$ best performance among all subsequences with a speedup $f\left(p_{v}, s_{j}\right)$ greater than 1 .

The pseudocode of the iterative compilation algorithm based on collaborative filtering is shown in Algorithm 2. As shown in Figure 4, the new target program $p_{\text {new }}$ is first transformed as an intermediate representation $I R_{\text {new }}^{0}$ without any optimizations. Then, the target program is further optimized with the iteratively recommended pass subsequences. Specifically, in the $l$ th iteration, the temporary optimized intermediate representation $I R^{l}$ is chosen as follows:

$$
I R_{\text {new }}^{l}=G\left(I R_{\text {new }}^{l-1}, \max _{s_{j} \in S_{n}} f\left(I R_{\text {new }}^{l-1}, s_{j}\right)\right),
$$

where $I R_{\text {new }}^{l-1}$ denotes the optimization result of the $(l-1)$-th iteration and $S_{n}$ denotes the subsequence set recommended in the $l$ th iteration. When the number of iteration arrives at a preset threshold $L$ or the program cannot be further optimized with the recommended pass subsequences, the iterative optimization process stops and it outputs the optimized program with the corresponding pass sequence.

4.2.3 Iterative Data Enhancement. As shown in Figure 4, the recommendation effectiveness in each iteration depends on not only a proper similarity metric but also sufficient program instances. However, the number of programs in most benchmarks and real applications is limited [12, 13]. To deal with this problem, we propose an iterative data enhancement method to generate new training programs based on a given set of programs.

A program can be defined as an instruction sequence with a given computer instruction set, for example, SSE2 and X86 [20]. At the instruction level, the optimized state of a program by a compiler pass or sequence can be regarded as a new instruction sequence, which means that it is a new program. Therefore, we use the Intermediate Representations (IRs) to represent the program as in [9] and treat a program optimized by one or more compiler passes as a new program. In this 


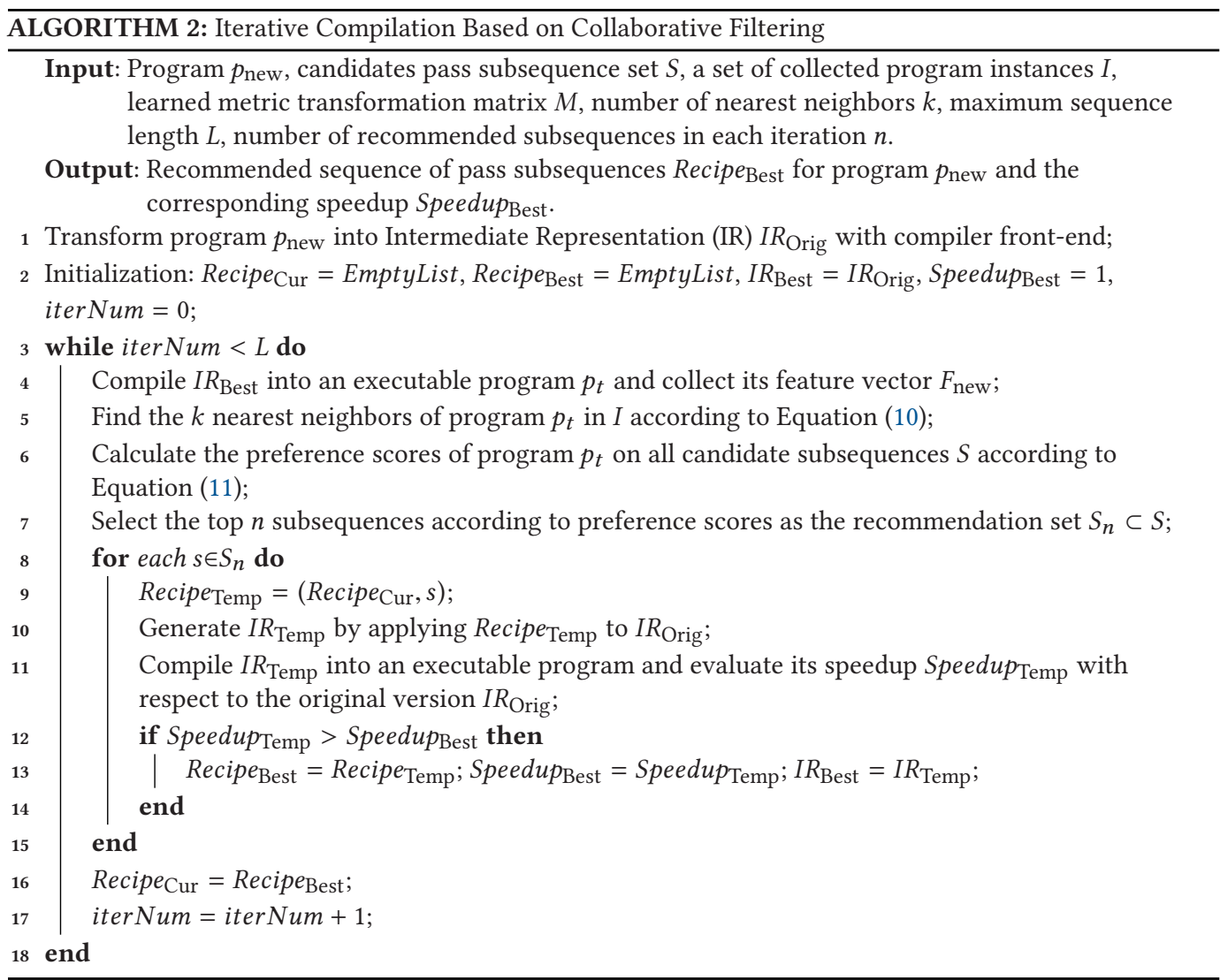

way, we can generate more training program instances based on a given set of programs and a set of compiler passes or subsequences.

As shown in Figure 5, each program instance is defined as an intermediate representation $I R$, and the intermediate representation of source program $p_{i}$ is denoted as $I R_{i}^{0}$. An intermediate representation $I R_{i}^{j}$ generated through applying a given pass sequence $s_{j}$ on $I R_{i}^{0}$ can be regarded as a new program instance. i.e.,

$$
I R_{i}^{j}=G\left(I R_{i}^{0}, s_{j}\right)
$$

In this way, we can iteratively generate more program instances, i.e., $I R_{i}^{(j, k)}=G\left(I R_{i}^{j}, s_{k}\right)$. These program instances can help us learn a more suitable similarity metric for the iterative collaborative recommendation with consideration of the combination effects of subsequences. For the newly generated program instances, dynamic program features are directly extracted from those IRs. Static features are extracted from the original source code (e.g., the root of the tree in Figure 5) and integrated with dynamic features to represent the generated program instances. Then these newly generated programs combined with the raw programs are used to collect training samples as usual, i.e., optimizing them with given pass sequences and evaluating the corresponding performance improvement.

Combined with the supervised pairwise metric learning method (Section 4.2.1), the proposed data enhancement method can help us to capture some high-order interactions among passes, i.e., 


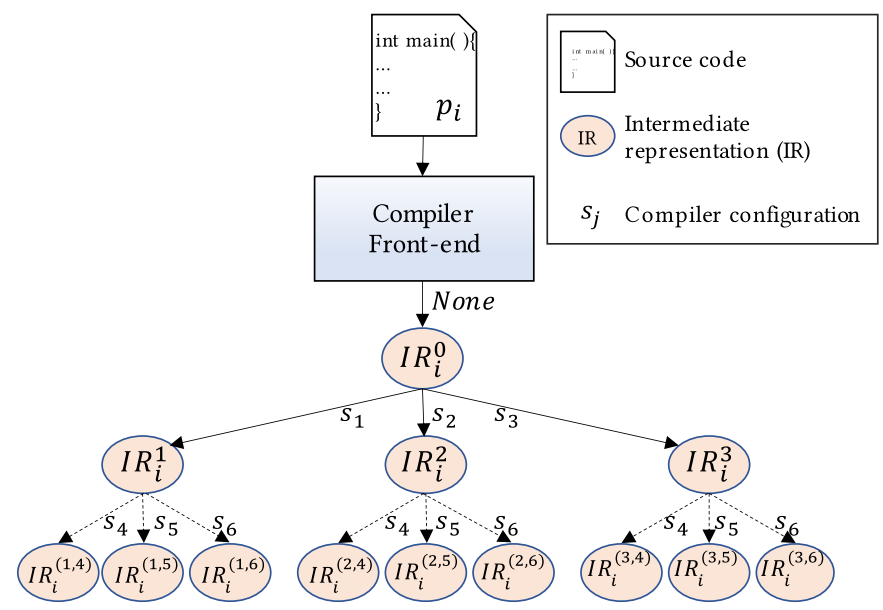

Fig. 5. Flowchart of the iterative data enhancement process. The Intermediate Representations (IR) of a program compiled with one or more subsequences are taken as new program samples.

the interactions between subsequences, which may be ignored during the phase of pass subsequence construction (Section 4.1).

\section{EXPERIMENTS}

In this section, we first introduce the experimental setup and evaluation methodology in Section 5.1. Then, the performance of our pass subsequence construction method and iterative compilation method are evaluated and compared with several state-of-the-art methods in Section 5.2 and 5.3, respectively. Last, we employ a series of experiments to evaluate the effectiveness of module design and the effects of parameter settings in Section 5.4 and 5.5, respectively.

\subsection{Experimental Setup}

5.1.1 Dataset and Experiment Platform. The full set of programs from the cTuning cBench benchmark v1.1 [21] are used as the experimental data, which contains 32 different programs ranging from automotive, security, and office sectors to telecommunications.

Experiments are run on a quad-core Intel(R) CPU i7-3630QM with Linux Ubuntu 16.4 OS. LLVM is adopted as the compiler. LLVM-clang and LLVM-link are used as the front-end and back-end, by which the source code is converted into an IR and the optimized IR is linked into an executable program, respectively. LLVM-opt is used as the optimization tool which apply an optimization sequence, such as -O3, on the IR. Since some of the passes provided by LLVM-opt are used for structural code analysis or code debugging, such as -deadarghaX0r, -view-cfg, and -transform-warning, we exclude these passes as they do not contribute to the performance improvement or even degrade the performance. In default, we use LLVM v3.8 as the compiler because several baseline methods like IS Pred. [3] and MiCOMP [4] used it and some results of these methods depend on it. With consideration of the rapid development of LLVM, we also do a comparison experiment using the latest LLVM, i.e., LLVM v12.0, in Section 5.3.

The execution time of each program is estimated by the time tool provided by Linux OS. A PIN-based dynamic instrumentation framework, called MICA [27], is used to characterize the dynamical behavior of a target program while executing. A plugin tool based on GCC, named Milepost-GCC [22, 23], is used to capture the static features of the source code without execution. 
A script written with Python language is used to automatically read, store, and analyze the raw data obtained by these tools.

5.1.2 Evaluation Metric and Methodology. To evaluate the empirical performance of different methods, we adopt the average speedup on all benchmark programs as the evaluation metric. Specifically, we use the harmonic mean speedup as in [4] and [26].

The speedup of a compiler option (i.e., a pass sequence) $o_{j}$ on a program $p_{i}$ is defined as:

$$
\operatorname{speedup}\left(p_{i}, o_{j}\right)=\frac{t\left(p_{i}, o_{\text {base }}\right)}{t\left(p_{i}, o_{j}\right)},
$$

where $t\left(p_{i}, o_{\text {base }}\right)$ denotes the execution time of program $p_{i}$ optimized by the base compiler option, e.g., - $\mathrm{O} 3$, and $t\left(p_{i}, o_{j}\right)$ denotes the execution time of program $p_{i}$ optimized by the compiler option $o_{j}$. The default dataset setting of cBench is used as the input for each program. With consideration of the execution time and measurement bias, we adjust the number of execution repetitions for each evaluation to make the measurement results stable and low-overhead.

All studies are cross-validated. Leave-one-out cross-validation (LOOCV) is adopted to better evaluate the performance of different methods as in [3], [4], and [11]. Each time, one program $p_{i}$ of the given program set $P$ is used as the test data, while the other programs are put together to form a training set. The process is repeated $|P|$ times with each program $p_{i} \in P$ used exactly once as the test data. The experimental results are recorded as the harmonic mean of the $|P|$ runs, i.e.,

$$
\text { speedup_Hmean }=\frac{|P|}{\sum_{p_{i} \in P} \frac{1}{\operatorname{speedup(p_{i})}} .}
$$

\subsection{Performance Evaluation of Pass Subsequence Construction}

The goal of this experiment is to evaluate the performance of the proposed pass subsequence construction method which is presented in Section 4.1.

5.2.1 Experimental Design. Two subsequence construction methods are used as baseline methods, including IS Pred. subsequences [3] and MiCOMP subsequences [4].

- IS Pred. subsequences [3] are fragments from the LLVM -O3 standard sequence with carefully considered collaborations among passes.

- MiCOMP subsequences [4] are automatically derived from the LLVM -O3 standard sequence using a hierarchical clustering method with consideration of the ordering information in the standard sequence.

In this experiment, we use an exhaustive search to evaluate the effectiveness of different subsequence construction methods, i.e., completely exploring the whole search space to determine the global optimal configuration of each subsequence group for each program with a given length of configuration. The harmonic mean speedups of all programs with various lengths of configuration are recorded. To ensure a fair comparison, we only use the passes in the LLVM standard level -O3 in this experiment. We construct five pass subsequences using the proposed method (Algorithm 1).

5.2.2 Experimental Results. Figure 6 shows the performance of different subsequence construction methods with various lengths. The speedups of different methods increase as the length of the sequence increases. However, the search space increases exponentially as the length of the sequence increases.

With different lengths of sequences, our data-driven method consistently performs better than both IS Pred. and MiCOMP. This result demonstrates the effectiveness of our data-driven subsequence construction method. In addition, the results compared with the subsequences designed 


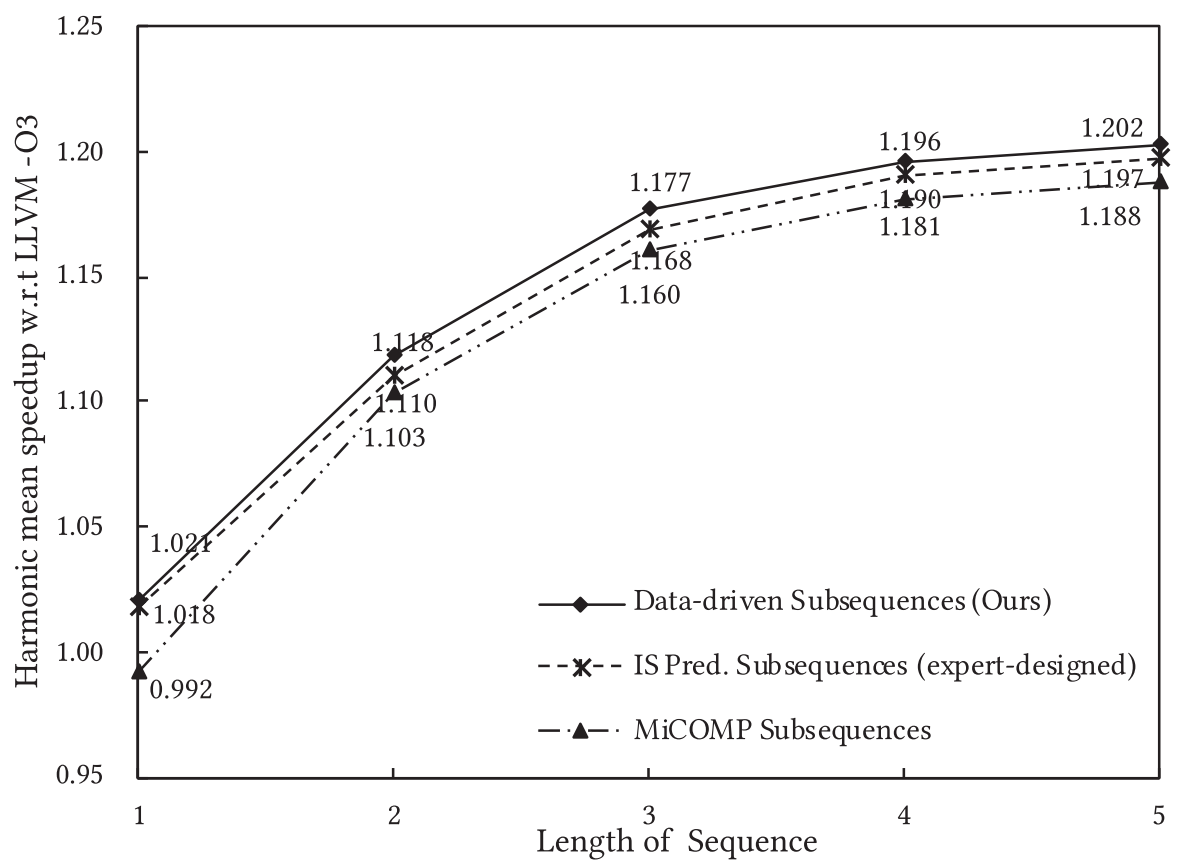

Fig. 6. Harmonic mean speedup of different subsequence construction methods using exhaustive search with various lengths of sequences.

by experts in IS Pred. show that our data-driven method can find some collaborative interactions that were ignored by the experts.

To further analyze the effectiveness of the constructed pass subsequences, we evaluate the performance of individual pass subsequences on all the 32 programs. As shown in Table 1, even using the constructed pass subsequences individually, programs can be optimized well and the best performance improvement can achieve 1.106. It is worth mentioning that these results are better than the results in Figure 6 with the length set as 1, because each of these subsequences can get better performance on its 31 training programs than on the one test program that was left out. Three passes, -mem 2 reg, -dse, and -correlated - propagation, appear in all the five best subsequences. Besides, we can find some characteristics of and interactions between the passes from these subsequences. For example, as a hardware independent pass, -sroa is used as the first pass in four of the five best subsequences, because it is used to do scalar replacement of aggregates, which seems to not dependent on other passes. After applying -sroa, some variables become dead stores, then the $-d s e$ pass, which intends to remove assignations of variables that are not read by any subsequent instructions, can be used to eliminate these stores. And after merging the same constant or the same variable with the pass -constmerge, the -correlated - propagation pass can be used to propagate values more effectively.

\subsection{Comparison with State-of-the-Art Methods}

The goal of this experiment is to evaluate the performance of the proposed iterative compilation method ICMC and make a comparison with several state-of-the-art compiler phase ordering methods.

5.3.1 Experimental Design. Five compiler phase ordering methods are used as baseline methods, including Focus Search [1], IS Pred. [3], MiCOMP [4], CF-TUNE [14], and CF-PS [11]. 
Table 1. The 5 Best Pass Subsequences and Their Harmonic Average Speedup with Respect to LLVM -O3

\begin{tabular}{|l|c|}
\hline Pass subsequences & Speedup \\
\hline $\begin{array}{l}\text {-mem2reg -correlated-propagation -scoped-noalias -globals-aa -lcssa } \\
\text {-jump-threading -tti -lazy-value-info -adce -dse -globalopt }\end{array}$ & 1.106 \\
\hline $\begin{array}{l}\text {-sroa -assumption-cache-tracker -dse -tailcallelim -constmerge } \\
\text {-correlated-propagation -inferattrs -mem2reg -argpromotion -ipsccp -loops }\end{array}$ & 1.106 \\
\hline $\begin{array}{l}\text {-sroa -dse -assumption-cache-tracker -tailcallelim -constmerge } \\
\text {-correlated-propagation -inferattrs -argpromotion -ipsccp -loops -mem2reg } \\
\text {-scoped-noalias }\end{array}$ & 1.104 \\
\hline $\begin{array}{l}\text {-sroa -assumption-cache-tracker -dse -constmerge -loop-vectorize } \\
\text {-lazy-value-info -argpromotion -correlated-propagation -globals-aa } \\
\text {-inferattrs -mem2reg -ipsccp -loops -scoped-noalias }\end{array}$ & 1.095 \\
\hline $\begin{array}{l}\text {-sroa -assumption-cache-tracker -dse -constmerge -correlated-propagation } \\
\text {-argpromotion -inferattrs -mem2reg -ipsccp -loops -scoped-noalias }\end{array}$ & 1.093 \\
\hline
\end{tabular}

- Focus Search [1] is a search-based compiler-phase ordering method that employs a Markov model to automatically search in those areas likely to achieve the best performance.

- Intermediate Speedup Predictor (IS Pred.)[3] is an iterative compilation method that tries to predict the next-best pass or subsequence iteratively with a learned linear regression model given the current state of the target program.

- MiCOMP [4] is a complete sequence prediction method that uses a regression model to directly predict the best whole sequence for a given program.

- CF-TUNE [14] is a complete sequence prediction method based on matrix factorization.

- CF-PS CF-PS [11] is a collaborative filtering-based method proposed for pass selection. We extend it for phase ordering by considering complete pass sequences rather than pass sets as the items to be recommended.

For Focus Search, we run a random search of 1,000 evaluations for each program with passes used in LLVM -O3 as offline training data as in [1]. Moreover, we use Laplace smoothing to avoid the sparse Markov transition matrix problem. For regression-based baselines, IS Pred. and MiCOMP, we set the parameter of PCA as $k=10$ to retain over $98 \%$ of the overall variance across all training data as in [3]. For CF-TUNE and CF-PS, we randomly choose 1,000 configurations composed of the subsequences used in MiCOMP [4] and IS Pred. [3], and other parameters are set as [14] and [11], respectively. Table 2 lists the parameter settings used in this experiment for our ICMC method. An aggregated pass subsequence set, which includes 5 subsequences constructed with passes used in LLVM -O3 and 5 subsequences constructed with passes not used in LLVM -O3 by Algorithm 1, is used in ICMC. To ensure a fair comparison, all methods are set to run the same number of evaluations to evaluate the performance of predicted pass sequences for each test program, which is set as 15 . For CF-TUNE, it requires another 15 evaluations for each test program to collect reaction features to make predictions.

5.3.2 Experimental Results. Table 3 shows the speedups of different methods with respect to the LLVM standard optimization level -O3. The proposed method ICMC gets the best performance on 29 out of the 32 programs compared with the baseline methods. In addition, it performs the same or better than LLVM -O3 on all the 32 programs. ICMC can reach an average speedup of 1.20 (up to 1.46) compared with the standard level -O3. On average, ICMC can achieve a performance 
Table 2. Parameter Settings of ICMC

\begin{tabular}{lc}
\hline Parameter & Value \\
\hline Number of kept effective passes $K$ in Algorithm 1 & 40 \\
Number of constructed subsequences $c$ & 5 \\
Number of nearest neighbors $k$ in Algorithm 2 & 5 \\
The maximum number of iterations $L$ & 5 \\
Number of recommended subsequences in each iteration $n$ & 3 \\
\hline
\end{tabular}

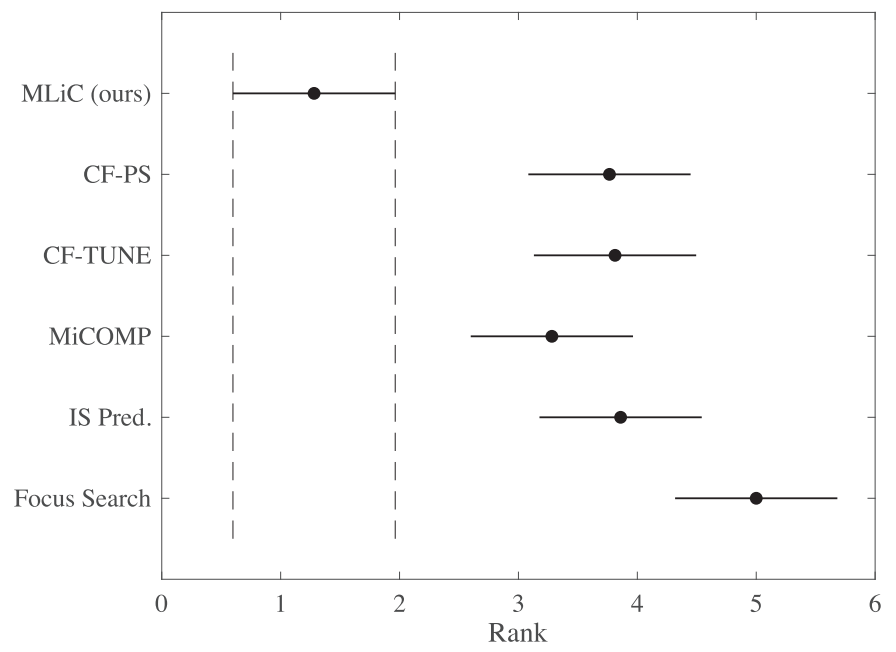

Fig. 7. Friedman test on performance speedup of different methods. The midpoint on each horizontal line indicates the average ranking of the corresponding method's performance. The horizontal line shows the confidence interval with the significance level of 0.05 in the Bonferroni test. If the horizontal lines of two methods do not overlap, it means that there is a significant difference between them.

improvement of $17.65 \%, 11.10 \%, 7.14 \%, 10.09 \%$, and 11.11\% compared with Focus Search, IS Pred. MiCOMP, CF-TUNE, and CF-PS, respectively.

To further compare the performance of different methods, we appeal to statistical tests. The Friedman test results are shown in Figure 7. ICMC achieves the best performance among all the compared methods. It is significantly better than all the other methods involved in the comparison. Among the compared methods, Focus Search performs the worst, as it uses only a transition probability model on the passes while ignoring the program-specific features. These results confirm that with proper program features, machine learning-based methods can attain better performance.

Table 4 shows the time cost of different methods in the training and the test stages. The time cost is measured by the number of evaluations. For each evaluation, we compile and run a training or test program to collect the performance information of compilation optimization, e.g., running time of the compiled program. Compared with the complete sequence prediction methods, e.g., MiCOMP, CF-TUNE and CF-PS, the iterative intermediate compilation methods such as IS Pred. and ICMC greatly shorten the training process. Even taking the subsequence construction utilized in the preprocessing phase into consideration, the running time of ICMC is still shorter than that of MiCOMP. It is worth mentioning that both the subsequence construction and the model training are done offline and once. Compared with the offline time consumption of these processes, we focus more on the online time consumption of model test and application. However, with the 
Table 3. Speedup of Different Methodswith Respect to LLVM -O3

\begin{tabular}{|c|c|c|c|c|c|c|}
\hline Applications & Focus Search & IS Pred. & MiCOMP & CF-TUNE & CF-PS & ICMC \\
\hline automotive_bitcount & 0.99 & 1.00 & 0.93 & 0.96 & 1.03 & 1.07 \\
\hline automotive_qsort1 & 0.89 & $\underline{0.92}$ & 0.90 & 0.89 & 0.91 & 1.04 \\
\hline automotive_susan_c & 1.10 & $\overline{1.19}$ & 1.33 & 1.32 & 1.36 & 1.38 \\
\hline automotive_susan_e & 1.06 & 1.13 & 1.32 & 1.27 & 1.13 & 1.35 \\
\hline automotive_susan_s & 1.12 & 1.18 & 1.16 & 1.06 & $\underline{1.20}$ & 1.23 \\
\hline bzip2d & $\underline{1.22}$ & 1.18 & 1.21 & 1.17 & 1.19 & 1.40 \\
\hline bzip2e & $\overline{1.02}$ & 1.08 & 1.28 & 1.10 & 1.20 & 1.28 \\
\hline consumer_jpeg_c & 1.01 & 1.17 & 1.31 & 1.15 & 1.16 & 1.37 \\
\hline consumer_jpeg_d & 1.06 & 1.20 & 1.20 & $\underline{1.30}$ & 1.23 & 1.35 \\
\hline consumer_mad & 1.05 & 1.10 & 1.03 & $\overline{1.05}$ & 1.08 & 1.12 \\
\hline consumer_lame & 1.10 & 1.13 & 1.18 & 1.11 & $\underline{1.19}$ & 1.22 \\
\hline consumer_tiff2bw & 1.33 & 1.37 & 1.37 & 1.36 & $\overline{1.27}$ & 1.34 \\
\hline consumer_tiff2rgba & 1.08 & $\overline{1.39}$ & $\overline{1.35}$ & 1.09 & 1.22 & 1.40 \\
\hline consumer_tiffdither & 1.03 & $\overline{1.30}$ & 1.23 & 1.31 & 1.22 & 1.38 \\
\hline consumer_tiffmedian & 1.07 & $\underline{1.41}$ & 1.39 & 1.18 & 1.25 & 1.45 \\
\hline network_dijkstra & 1.00 & $\underline{1.04}$ & 1.03 & 0.86 & 0.91 & 1.04 \\
\hline network_patricia & 0.96 & 0.92 & 1.01 & 1.03 & 0.86 & 1.05 \\
\hline office_ghostscript & 1.15 & 1.22 & 1.20 & $\overline{1.25}$ & 1.23 & 1.37 \\
\hline office_ispell & 1.01 & 1.00 & 1.04 & $\overline{1.09}$ & 1.11 & 1.15 \\
\hline office_rsynth & 0.99 & 0.93 & 1.04 & 1.01 & $\overline{0.94}$ & 1.05 \\
\hline office_stringsearch1 & 0.89 & 0.92 & $\overline{0.78}$ & 0.87 & 0.82 & 1.04 \\
\hline security_blowfish_d & 1.03 & 1.05 & $\underline{1.06}$ & 1.04 & 0.91 & 1.08 \\
\hline security_blowfish_e & 1.03 & 1.08 & 1.10 & 1.07 & 0.94 & 1.06 \\
\hline security_pgp_d & 0.98 & 1.02 & $\overline{1.05}$ & 1.12 & 1.08 & 1.33 \\
\hline security_pgp_e & 0.92 & 1.03 & 1.10 & 1.14 & $\underline{1.17}$ & 1.22 \\
\hline security_rijndael_d & 0.96 & 0.95 & 0.98 & 0.99 & $\overline{0.98}$ & 1.02 \\
\hline security_rijndael_e & 0.99 & 0.96 & 0.98 & 0.96 & 0.98 & 1.00 \\
\hline security_sha & 0.92 & $\underline{1.02}$ & 1.00 & 1.02 & 1.01 & 1.08 \\
\hline telecom_adpcm_c & 1.04 & $\overline{1.23}$ & 1.40 & $\overline{1.23}$ & 1.39 & 1.46 \\
\hline telecom_adpcm_d & 1.07 & 1.13 & 1.18 & 1.10 & 1.16 & 1.20 \\
\hline telecom_crc32 & 1.04 & 0.98 & $\overline{1.02}$ & 1.01 & 0.99 & 1.09 \\
\hline telecom_gsm & $\overline{0.92}$ & 0.87 & 1.24 & 1.24 & 1.17 & 1.21 \\
\hline Harmonic Mean & 1.02 & 1.08 & $\overline{1.12}$ & $\overline{1.09}$ & 1.08 & 1.20 \\
\hline \# of best perf. & $\mathbf{0}$ & 1 & 4 & 1 & $\mathbf{0}$ & 29 \\
\hline
\end{tabular}

The best results are bolded and the best baseline results are underlined. The last row denotes the number of best speedups of each method out of the 32 test applications.

same online time consumption, the offline time consumption determines the relative execution efficiency of different methods.

Considering the rapid development of LLVM, we also compare the proposed method ICMC with three baselines, including Focus Search [1], CF-TUNE [14] and CF-PS [11], using the latest LLVM v12.0. We simply use the last program of each sector in alphabetical order as test programs, including automotive_susan_s, bzip2e, consumer_tiffmedian, network_patricia, office_stringsearch1, security_sha and telecom_gsm. The other 25 programs are used as training programs. As shown in Table 5 , the proposed method ICMC performs the best on all seven test programs and achieves 
Table 4. Time Cost of Different Methods Measured by the Number of Evaluations (\# of Evals Per Program)

\begin{tabular}{|l|c|c|c|}
\hline Method & Subsequence construction & Training phase & Test phase \\
\hline Focus Search & - & 1000 & 15 \\
\hline IS Pred. & - & 4 & 15 \\
\hline MiCOMP & 0 & 3906 & 15 \\
\hline CF-TUNE & - & 1000 & $15+15$ \\
\hline CF-PS & - & 1000 & 15 \\
\hline ICMC & 3331 & 10 & 15 \\
\hline
\end{tabular}

Table 5. Speedup of Different Methods with Respect to -O3 Using LLVM v12.0

\begin{tabular}{|l|c|c|c|c|}
\hline Applications & Focus Search & CF-TUNE & CF-PS & ICMC \\
\hline automotive_susan_s & 1.09 & $\underline{1.20}$ & $\underline{1.20}$ & $\mathbf{1 . 2 6}$ \\
\hline bzip2e & 1.10 & $\underline{1.27}$ & 1.21 & $\mathbf{1 . 3 1}$ \\
\hline consumer_tiffmedian & 1.32 & 1.37 & $\underline{1.40}$ & $\mathbf{1 . 4 7}$ \\
\hline network_patricia & 0.92 & $\underline{0.94}$ & 0.85 & $\mathbf{1 . 0 6}$ \\
\hline office_stringsearch1 & 0.78 & 0.84 & $\underline{0.91}$ & $\mathbf{1 . 0 1}$ \\
\hline security_sha & 0.90 & $\underline{1.06}$ & 1.03 & $\mathbf{1 . 1 2}$ \\
\hline telecom_gsm & 0.95 & 1.18 & $\underline{1.22}$ & $\mathbf{1 . 2 8}$ \\
\hline
\end{tabular}

a performance improvement of $21.76 \%, 9.43 \%$ and $10.18 \%$ compared with Focus Search, CF-TUNE and CF-PS, respectively, on average.

\subsection{Ablation Studies}

The goal of this experiment is to evaluate the effectiveness of module design of ICMC.

5.4.1 Experimental Design. There are several key modules in ICMC, including pass subsequence construction, metric learning, data collection, and enhancement. To evaluate the effectiveness of these modules, we replace them with some traditional methods and compare the performance of ICMC before and after the replacement. For pass subsequence construction, we try to replace our data-driven method with MiCOMP's method. For metric learning, we try to replace the supervised metric learning method with traditional PCA. For data collection and enhancement, we try to compare the performance of ICMC with and without data enhancement on different feature sets.

To evaluate the relative effectiveness of different methods, we define a metric, called improvement ratio, to measure the relative effectiveness of a method $B$ over another method $A$ on a program $p$ as follows:

$$
\text { improvement_ratio }(p, A, B)=\frac{t(p, A)}{t(p, B)},
$$

where $t(p, A)$ and $t(p, B)$ denote the execution time of the program $p$ optimized by method $A$ and method $B$, respectively. Improvement_ratio $(p, A, B)>1$ means that method $B$ is more effective than method $A$ for program $p$.

5.4.2 Experimental Results. Figure 8 shows the effectiveness of subsequence construction and metric learning of ICMC. Compared with unsupervised PCA, the supervised metric learning method is more effective when combined with different pass subsequence sets. It confirms that the supervised metric learning method can help to capture the preference similarity between 


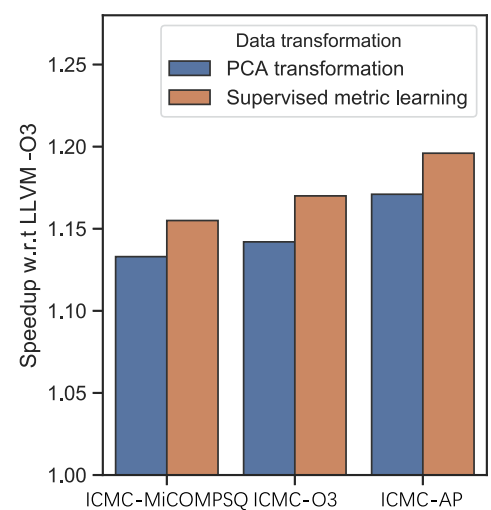

(a)

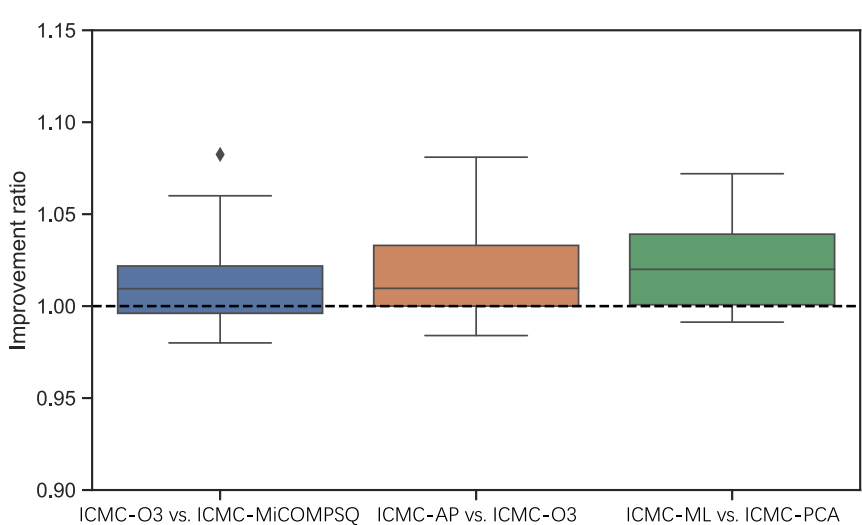

(b)

Fig. 8. Effectiveness of subsequence construction and supervised metric learning. (a) Harmonic mean speedup with different module settings with respect to LLVM -O3. (b) Improvement ratios of the proposed modules compared with traditional methods. ICMC-MiCOMPSQ denotes ICMC with subsequences replaced by MiCOMP's subsequences. ICMC-O3 denotes ICMC with only passes in -O3. ICMC-AP denotes ICMC with all available passes. ICMC-ML denotes ICMC with the supervised metric learning method. ICMCPCA denotes ICMC with metric learning replaced by traditional PCA.

programs while mitigating the effects of redundant and irrelevant features for compilation optimization. Compared with MiCOMP's subsequence construction method, our subsequence construction method is more effective with only the passes in the standard -O3 sequence. It shows the ability of our data-driven method to capture the collaborations among passes. In addition, our datadriven method can make use of passes not contained in the -O3 sequence. Experimental results show that it can significantly improve the performance of ICMC with the help of these passes on most programs. This finding confirms that the use of extra passes can provide more useful optimization options for different code segments and produce better optimization effects.

Figure 9 shows the effectiveness of data collection and enhancement of ICMC. Compared with using only dynamic program features, the adding of static code features can improve the performance of iterative compilation. With the help of the proposed data enhancement method, we can significantly improve the performance of ICMC.

\subsection{Effects of Different Parameter Settings}

The goal of this experiment is to evaluate the effects of different parameter settings on the performance of ICMC.

5.5.1 Experimental Design. There are several key parameters for ICMC, including the number of passes retained to construct subsequences $K$, the number of constructed pass subsequences $c$, the number of nearest neighbors used for personalized recommendation $k$, the length of the recommendation list in each iteration $n$, and the maximum number of iterations $L$, i.e., the maximum length of sequences. We evaluate the effects of different settings of these parameters on the performance of ICMC.

5.5.2 Experimental Results. Figure 10 shows the effects of different settings of the number of retained passes $K$ and the number of constructed subsequences $c$. It demonstrates that the performance of ICMC is relatively stable when the values of $K$ and $c$ are not too small or too large. When the value of $K$, i.e., the number of passes used to construct subsequences, is too small, ICMC yields 


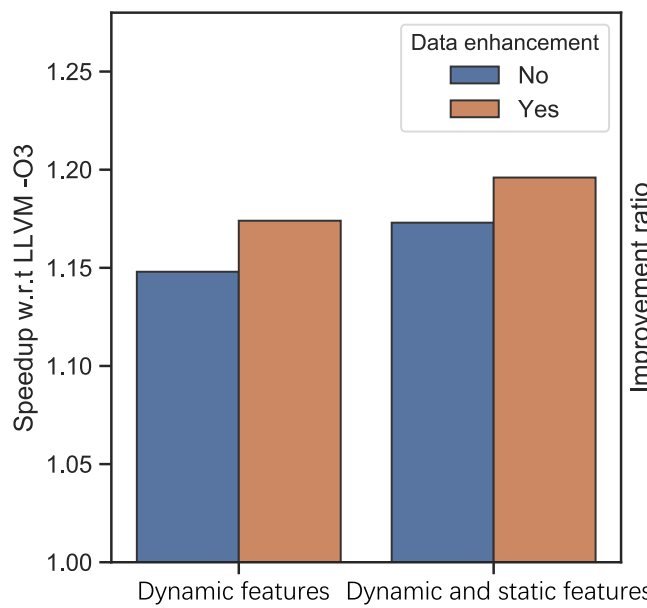

(a)

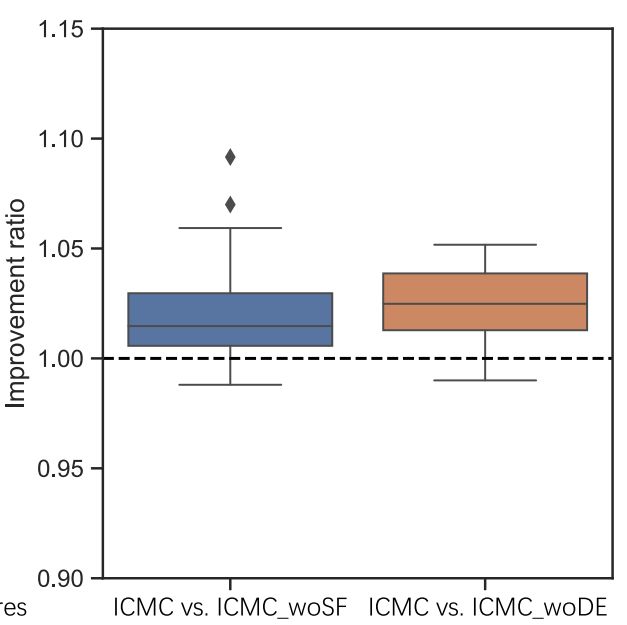

(b)

Fig. 9. Effectiveness of data collection (feature choices) and enhancement. (a) Harmonic mean speedup with different module settings with respect to LLVM -O3. (b) Improvement ratios of the proposed modules compared with traditional methods. ICMC-woSF denotes ICMC without using static code-features. ICMC-woDE denotes ICMC without using data enhancement.

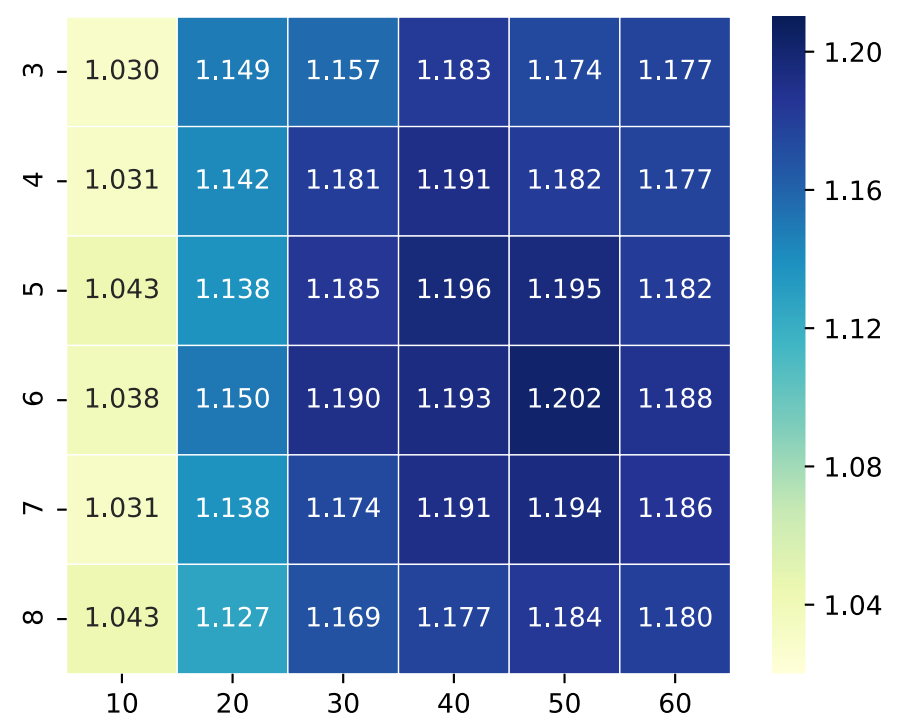

Fig. 10. The effects of different settings of the number of retained passes $K$ (rows) and the number of constructed subsequences $c$ (columns).

a poor performance for ignoring many useful passes. In consideration of both performance and complexity, we suggest to set $K=40$ and $c=5$.

Table 6 shows the effects of different settings of the neighborhood size $k$ and the length of the recommendation list in each iteration $n$. As the value of $n$ increases, the performance of ICMC first increases significantly and then remains stable. The effect of parameter $k$ is similar. In consideration of both performance and complexity, we suggest to set $k=5$ and $n=3$. 
Table 6. The Effects of Different Settings of the Neighborhood Size $k$ and the Length of the Recommendation List in Each Iteration $n$

\begin{tabular}{|l|l|l|l|l|l|}
\hline & $n=1$ & $n=2$ & $n=3$ & $n=4$ & $n=5$ \\
\hline$k=3$ & 1.131 & 1.149 & 1.172 & 1.171 & 1.190 \\
\hline$k=5$ & 1.140 & 1.170 & 1.196 & 1.204 & 1.199 \\
\hline$k=7$ & 1.148 & 1.163 & 1.196 & 1.199 & 1.195 \\
\hline$k=9$ & 1.148 & 1.166 & 1.183 & 1.181 & 1.188 \\
\hline
\end{tabular}

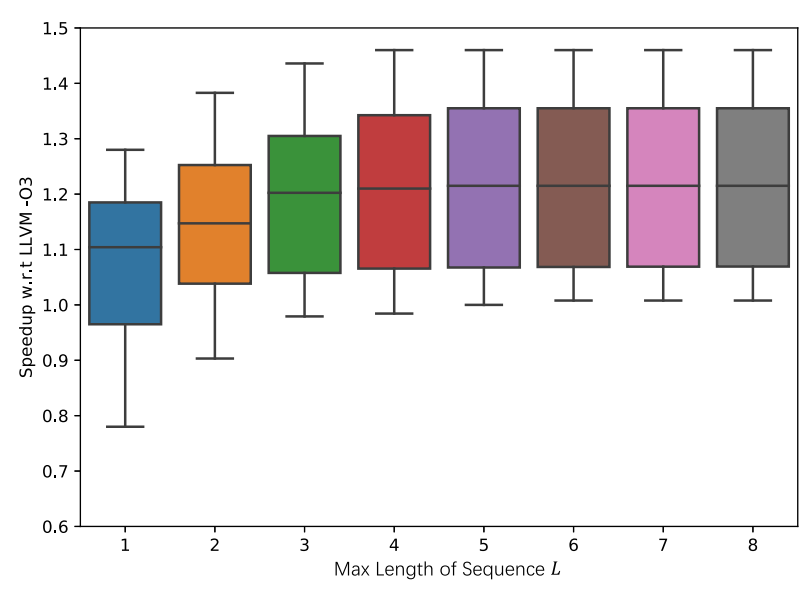

Fig. 11. Effect of the max length of the sequence $L$.

Figure 11 shows the performance of ICMC with various maximum numbers of iterations $L$, i.e., the maximum length of sequences. As the value of $L$ increases, the performance of ICMC first increases and then tends to be stable. In consideration of both performance and complexity, we suggest to set $L=5$.

\section{CONCLUSIONS}

To address the compiler pass selection and phase ordering problem, which is an NP-hard problem, we first proposed a data-driven subsequence construction method. It can not only prune the search space but can also make use of all available passes besides those not contained in standard compiler levels. Then, we proposed an iterative compilation method based on collaborative filtering and metric learning, which can effectively find some suitable pass sequences for a new program with a few compilations and evaluations.

In this article, we only took the reduction of execution time as the objective. However, there are several other objectives in real applications, such as the reduction of the code size or energy consumption. In the future, we will evaluate the performance of ICMC with these objectives and study the multi-objective compiler auto-tuning problem [19]. Furthermore, during the construction of the pass subsequence, we focused on the pairwise dependency among passes. In the future, we will mine more complex and high-order dependency among passes. In addition, since we only used the program features extracted by two existing tools, some useful information for our task may have been overlooked. Therefore, in the future, we will try to directly learn features from program source codes or intermediate representations [17, 34]. 


\section{REFERENCES}

[1] Felix Agakov, Edwin Bonilla, John Cavazos, Bjorn Franke, Grigori Fursin, Michael O’Boyle, John Thomson, Marc Toussaint, and Christopher K. I. Williams. 2006. Using machine learning to focus iterative optimization. In Proceedings of the International Symposium on Code Generation and Optimization (CGO'06). IEEE, 295-305.

[2] L. Almagor, Keith D. Cooper, Alexander Grosul, Timothy J. Harvey, Steven W. Reeves, Devika Subramanian, Linda Torczon, and Todd Waterman. 2004. Finding effective compilation sequences. In Proceedings of the 2004 ACM SIGPLAN/SIGBED Conference on Languages, Compilers, and Tools for Embedded Systems (Washington, DC) (LCTES'04) (Washington, DC). ACM, 231-239. https://doi.org/10.1145/997163.997196

[3] Amir H. Ashouri, Andrea Bignoli, Gianluca Palermo, and Cristina Silvano. 2016. Predictive modeling methodology for compiler phase-ordering. In Proceedings of the 7th Workshop on Parallel Programming and Run-Time Management Techniques for Many-core Architectures and the 5th Workshop on Design Tools and Architectures For Multicore Embedded Computing Platforms (Prague, Czech Republic) (PARMA-DITAM'16). ACM, New York, 7-12.

[4] Amir H. Ashouri, Andrea Bignoli, Gianluca Palermo, Cristina Silvano, Sameer Kulkarni, and John Cavazos. 2017. MiCOMP: Mitigating the compiler phase-ordering problem using optimization sub-sequences and machine learning. ACM Transactions on Architecture and Code Optimization 14, 3, Article 29 (Sept. 2017), 28 pages. https://doi.org/10. 1145/3124452

[5] Amir H. Ashouri, William Killian, John Cavazos, Gianluca Palermo, and Cristina Silvano. 2018. A survey on compiler autotuning using machine learning. ACM Computing Survey 51, 5, Article 96 (Sept. 2018), 42 pages. https://doi.org/ $10.1145 / 3197978$

[6] Amir H. Ashouri, Giovanni Mariani, Gianluca Palermo, Eunjung Park, John Cavazos, and Cristina Silvano. 2016. COBAYN: Compiler autotuning framework using Bayesian networks. ACM Transactions on Architecture and Code Optimization 13, 2, Article 21 (June 2016), 25 pages. https://doi.org/10.1145/2928270

[7] Amir H. Ashouri, Giovanni Mariani, Gianluca Palermo, and Cristina Silvano. 2014. A Bayesian network approach for compiler auto-tuning for embedded processors. In Proceedings of the IEEE 12th Symposium on Embedded Systems for Real-time Multimedia (Greater Noida, India) (ESTIMedia'14). IEEE, 90-97. https://doi.org/10.1109/ESTIMedia.2014. 6962349

[8] Inpyo Bae, Barend Harris, Hyemi Min, and Bernhard Egger. 2018. Auto-tuning CNNs for coarse-grained reconfigurable array-based accelerators. IEEE Transactions on Computer-Aided Design of Integrated Circuits and Systems 37, 11 (2018), 2301-2310. https://doi.org/10.1109/TCAD.2018.2857278

[9] Tal Ben-Nun, Alice Shoshana Jakobovits, and Torsten Hoefler. 2018. Neural code comprehension: A learnable representation of code semantics. In Advances in Neural Information Processing Systems 31 (NIPS'18). Curran Associates, Inc., 3585-3597.

[10] John Cavazos, Grigori Fursin, Felix Agakov, Edwin Bonilla, Michael F. P. O’Boyle, and Olivier Temam. 2007. Rapidly selecting good compiler optimizations using performance counters. In Proceedings of the International Symposium on Code Generation and Optimization (CGO'07). IEEE, 185-197. https://doi.org/10.1109/CGO.2007.32

[11] Stefano Cereda, Gianluca Palermo, Paolo Cremonesi, and Stefano Doni. 2020. A collaborative filtering approach for the automatic tuning of compiler optimisations. In Proceedings of the 21st ACM SIGPLAN/SIGBED Conference on Languages, Compilers, and Tools for Embedded Systems (LCTES'20) (London, United Kingdom). ACM, New York, 15-25. https://doi.org/10.1145/3372799.3394361

[12] Yang Chen, Yuanjie Huang, Lieven Eeckhout, Grigori Fursin, Liang Peng, Olivier Temam, and Chengyong Wu. 2010. Evaluating iterative optimization across 1000 datasets. In Proceedings of the 31st ACM SIGPLAN Conference on Programming Language Design and Implementation (PLDI'10) (Toronto, Ontario, Canada). ACM, New York, 448-459. https://doi.org/10.1145/1806596.1806647

[13] Alton Chiu, Joseph Garvey, and Tarek S. Abdelrahman. 2015. Genesis: A language for generating synthetic training programs for machine learning. In Proceedings of the 12th ACM International Conference on Computing Frontiers (CF'15) (Ischia, Italy). ACM, New York, Article 8, 8 pages. https://doi.org/10.1145/2742854.2742883

[14] Eleftherios-Iordanis Christoforidis, Sotirios Xydis, and Dimitrios Soudris. 2018. CF-TUNE: Collaborative filtering auto-tuning for energy efficient many-core processors. IEEE Computer Architecture Letters 17, 1 (2018), 25-28.

[15] Keith D. Cooper, Philip J. Schielke, and Devika Subramanian. 1999. Optimizing for reduced code space using genetic algorithms. In Proceedings of the ACM SIGPLAN 1999 Workshop on Languages, Compilers, and Tools for Embedded Systems (LCTES'99) (Atlanta, Georgia). ACM, New York, 1-9. https://doi.org/10.1145/314403.314414

[16] Keith D. Cooper, Devika Subramanian, and Linda Torczon. 2002. Adaptive optimizing compilers for the 21st century. The fournal of Supercomputing 23, 1 (2002), 7-22. https://doi.org/10.1023/A:1015729001611

[17] Chris Cummins, Pavlos Petoumenos, Zheng Wang, and Hugh Leather. 2017. End-to-end deep learning of optimization heuristics. In Proceedings of the 26th International Conference on Parallel Architectures and Compilation Techniques (PACT'17) (Portland, OR). IEEE, 219-232. https://doi.org/10.1109/PACT.2017.24 
[18] Chris Cummins, Pavlos Petoumenos, Zheng Wang, and Hugh Leather. 2017. Synthesizing benchmarks for predictive modeling. In Proceedings of the 2017 International Symposium on Code Generation and Optimization (CGO'17) (Austin, TX). IEEE, 86-99. https://doi.org/10.1109/CGO.2017.7863731

[19] Yi Ding, Nikita Mishra, and Henry Hoffmann. 2019. Generative and multi-phase learning for computer systems optimization. In Proceedings of the 46th International Symposium on Computer Architecture (ISCA'19) (Phoenix, AZ) . ACM, New York, 39-52. https://doi.org/10.1145/3307650.3326633

[20] Yunsi Fei and Z. Jerry Shi. 2007. Microarchitectural support for program code integrity monitoring in applicationspecific instruction set processors. In Proceedings of the Conference on Design, Automation and Test in Europe (DATE’07) (Nice, France). EDA Consortium, San Jose, CA, 815-820.

[21] Grigori Fursin, John Cavazos, and Olivier Temam. 2007. Midatasets: Creating the conditions for a more realistic evaluation of iterative optimization. In Proceedings of the 2nd International Conference on High Performance Embedded Architectures and Compilers (HiPEAC'07) (Ghent, Belgium). Springer-Verlag, Berlin, 245-260.

[22] Grigori Fursin, Yuriy Kashnikov, Abdul W. Memon, Zbigniew Chamski, Olivier Temam, Mircea Namolaru, Elad Yomtov, Bilha Mendelson, Ayal Zaks, Eric Courtois, Francois Bodin, Phil Barnard, Elton Ashton, Edwin Bonilla, John Thomson, Christopher K. I. Williams, and Michael O’Boyle. 2011. Milepost GCC: Machine learning enabled selftuning compiler. International fournal of Parallel Programming 39, 3 (2011), 296-327. https://doi.org/10.1007/s10766010-0161-2

[23] Grigori Fursin, Anton Lokhmotov, and Ed Plowman. 2016. Collective knowledge: Towards R\&D sustainability. In Proceedings of the 2016 Conference on Design, Automation and Test in Europe (DATE'16) (Ghent, Belgium). EDA Consortium, San Jose, CA, 864-869.

[24] Kyriakos Georgiou, Craig Blackmore, Samuel Xavier-de-Souza, and Kerstin Eder. 2018. Less is more: Exploiting the standard compiler optimization levels for better performance and energy consumption. In Proceedings of the 21st International Workshop on Software and Compilers for Embedded Systems (SCOPES'18) (Sankt Goar, Germany). ACM, New York, 35-42. https://doi.org/10.1145/3207719.3207727

[25] Felix J. Herrmann, Urs Büniger, and Dirk J. Verschuur. 2007. Non-linear primary-multiple separation with directional curvelet frames. Geophysical Journal International 170, 2 (2007), 781-799. https://doi.org/10.1111/j.1365-246X.2007. 03360.x

[26] Torsten Hoefler and Roberto Belli. 2015. Scientific benchmarking of parallel computing systems: Twelve ways to tell the masses when reporting performance results. In Proceedings of the International Conference for High Performance Computing, Networking, Storage and Analysis (SC'15) (Austin, Texas). ACM, New York, Article 73, 12 pages.

[27] Kenneth Hoste and Lieven Eeckhout. 2007. Microarchitecture-independent workload characterization. IEEE Micro 27, 3 (2007), 63-72. https://doi.org/10.1109/MM.2007.56

[28] Michael R. Jantz and Prasad A. Kulkarni. 2013. Exploiting phase inter-dependencies for faster iterative compiler optimization phase order searches. In Proceedings of the 2013 International Conference on Compilers, Architectures and Synthesis for Embedded Systems (CASES'13) (Montreal, Quebec, Canada). IEEE, Article 7, 10 pages.

[29] Toru Kisuki, Peter M. W. Knijnenburg, Michael F. P. O’Boyle, and Harry A. G. Wijshoff. 2000. Iterative compilation in program optimization. In Proceedings of the 15th Workshop on Compilers for Parallel Computers (CPC'10). SpringerVerlag, Berlin, 35-44.

[30] Prasad Kulkarni, Stephen Hines, Jason Hiser, David Whalley, Jack Davidson, and Douglas Jones. 2004. Fast searches for effective optimization phase sequences. ACM SIGPLAN Notices 39, 6 (June 2004), 171-182.

[31] Prasad A. Kulkarni, David B. Whalley, Gary S. Tyson, and Jack W. Davidson. 2006. Exhaustive optimization phase order space exploration. In Proceedings of the International Symposium on Code Generation and Optimization (CGO'06) IEEE, 306-318. https://doi.org/10.1109/CGO.2006.15

[32] Sameer Kulkarni and John Cavazos. 2012. Mitigating the compiler optimization phase-ordering problem using machine learning. ACM SIGPLAN Notices 47, 10 (Oct. 2012), 147-162. https://doi.org/10.1145/2398857.2384628

[33] Hugh Leather, Edwin Bonilla, and Michael O’Boyle. 2009. Automatic feature generation for machine learning based optimizing compilation. In Proceedings of the 7th Annual IEEE/ACM International Symposium on Code Generation and Optimization (CGO'09). IEEE, 81-91. https://doi.org/10.1109/CGO.2009.21

[34] Hugh Leather, Edwin Bonilla, and Michael O’Boyle. 2014. Automatic feature generation for machine learning-based optimising compilation. ACM Transactions on Architecture and Code Optimization 11, 1, Article 14 (Feb. 2014), 32 pages. https://doi.org/10.1145/2536688

[35] Bo Liu, Ying Wei, Yu Zhang, and Qiang Yang. 2017. Deep neural networks for high dimension, low sample size data. In Proceedings of the 26th International foint Conference on Artificial Intelligence (IFCAI'17). 2287-2293.

[36] Eric Y. Liu, Zhishan Guo, Xiang Zhang, Vladimir Jojic, and Wei Wang. 2012. Metric learning from relative comparisons by minimizing squared residual. In Proceedings of the 12th IEEE International Conference on Data Mining (ICDM’12) (Brussels, Belgium). IEEE, 978-983. https://doi.org/10.1109/ICDM.2012.38 
[37] Luiz G. A. Martins, Ricardo Nobre, Alexandre C. B. Delbem, Eduardo Marques, and João M. P. Cardoso. 2014. Exploration of compiler optimization sequences using clustering-based selection. In Proceedings of the 2014 SIGPLAN/SIGBED Conference on Languages, Compilers and Tools for Embedded Systems (LCTES'14) (Edinburgh, United Kingdom). ACM, New York, 63-72.

[38] Amini Mehdi, Coelho Fabien, Irigoin François, and Keryell Ronan. 2011. Static compilation analysis for hostaccelerator communication optimization. In Proceedings of the 24th International Workshop onLanguages and Compilers for Parallel Computing (LCPC'11), (Fort Collins, CO). Springer, Berlin, 237-251.

[39] Eunjung Park, John Cavazos, and Marco A. Alvarez. 2012. Using graph-based program characterization for predictive modeling. In Proceedings of the 10th International Symposium on Code Generation and Optimization (CGO'12) (San Jose, CA) . ACM, New York, 196-206. https://doi.org/10.1145/2259016.2259042

[40] Eunjung Park, John Cavazos, Louis-Noël Pouchet, Cédric Bastoul, Albert Cohen, and Ponnuswamy Sadayappan. 2013. Predictive modeling in a polyhedral optimization space. International fournal of Parallel Programming 41, 5 (2013), 704-750. https://doi.org/10.1007/s10766-013-0241-1

[41] Suresh Purini and Lakshya Jain. 2013. Finding good optimization sequences covering program space. ACM Transactions on Architecture and Code Optimization 9, 4, Article 56 (Jan. 2013), 23 pages.

[42] Mark Stephenson, Saman Amarasinghe, Martin Martin, and Una-May O'Reilly. 2003. Meta optimization: Improving compiler heuristics with machine learning. In Proceedings of the ACM SIGPLAN 2003 Conference on Programming Language Design and Implementation (San Diego, CA) (PLDI'03). ACM, New York, 77-90.

[43] Michel Steuwer, Toomas Remmelg, and Christophe Dubach. 2016. Matrix multiplication beyond auto-tuning: Rewrite-based GPU code generation. In Proceedings of the International Conference on Compilers, Architectures and Synthesis for Embedded Systems (CASES'16) (Pittsburgh, PA). ACM, New York, Article 15, 10 pages. https://doi.org/10.1145/2968455.2968521

[44] John Thomson, Michael O’Boyle, Grigori Fursin, and Björn Franke. 2009. Reducing training time in a one-shot machine learning-based compiler. In Proceedings of the 22nd International Conference on Languages and Compilers for Parallel Computing (LCPC'09) (Newark DE). Springer-Verlag, Berlin, 399-407. https://doi.org/10.1007/978-3-64213374-9_28

[45] Zheng Wang and Michael O’Boyle. 2018. Machine learning in compiler optimization. Proceedings of the IEEE 106, 11 (Nov 2018), 1879-1901. https://doi.org/10.1109/JPROC.2018.2817118

[46] Stella Yu and Jianbo Shi. 2003. Multiclass spectral clustering. In Proceedings of the 9th IEEE International Conference on Computer Vision (ICCV'03) (Nice, France). IEEE, 313-319. https://doi.org/10.1109/ICCV.2003.1238361

Received December 2020; revised July 2021; accepted August 2021 